\title{
Relationship between Aerobic Exercise and S-Klotho in Aging
}

\author{
Moran Saghiv ${ }^{1 *}$ and Michael Sagiv ${ }^{2}$ \\ ${ }^{1}$ Department of Kinesiology, North Carolina, USA \\ ${ }^{2}$ Department of Life Sciences, Israel, USA
}

*Corresponding author: Moran Saghiv, Department of Kinesiology, NC A\&T State University, USA.

Received Date: April 10, 2019

Published Date: April 25, 2019

\begin{abstract}
Aging causes structural and functional decrements in skeletal muscles (sarcopenia), bone mineral density, cardiovascular and oxygen uptake. Progress of many cardiovascular diseases as atherosclerosis, endothelial dysfunction and hypertension with aging. This points out that aging should be seen as a risk factor. This is causes an imbalance between vasodilator-vasoconstriction substances ratio formed by the endothelium resulting in a significant reduction in nitric oxide production. Nitric oxide decrement causes some anomalies in blood artery function along, with an increase in oxidative stress molecules production, which in turn increase production of ROS and nitrogen species. Secreted klotho regulates nitric oxide production which in turn benefits endothelial function: membrane klotho acts through (FGF)-23, while secreted klotho regulates nitric oxide produced in the endothelium. Exercise inactivity accelerates aging and its consequences, it is suggested as a major reason for increased disease and mortality. In recent years however, there are enough studies about the effect of aerobic exercise on blood circulating s-Klotho. Recently, it has been demonstrated that the response of circulating s-klotho depends on aerobic fitness level: values of s-Klotho were significantly higher in trained individuals compared to untrained once, suggesting that aerobic exercise training is a suitable model for mechanistically probing the role of physical activity on s-Klotho expression. In conclusions, the present review suggests that aerobic exercise delays aging process by increase Klotho gene expression, which in turn, reduces ROS damages to the cell. The relationships between the circulating s-Klotho levels a potential anti-aging factor and aerobic exercise are beneficial to physically active aged individuals. Endothelial dysfunction improvement depends on increased circulating s-klotho levels through aerobic exercise. The purpose of this review is to discuss factors such as, oxidative stress and pro-inflammatory cytokines involved in the progress of endothelial dysfunction due to the aging process, with impacts related to, s-Klotho and aerobic exercise on the endothelial dysfunction process.
\end{abstract}

Keywords: Nitric oxide; Arteriosclerosis; Hypertension; Apoptosis; Oxidative stress; FGF23

\section{Aging considerations}

Aging is a usual life course that includes all physiological systems, seen as an advanced biological degeneration and decrease in physical capacities that raises the possibility of disease. Aging is a gradual decline of various organ functions of the body, deteriorating phenomenon defined by relations between the genetic expressions and external factors [1]. Ecological epigenetics defines how natural factors affect cellular epigenetics and, thus, human performance [2]. Aging is a multifaceted process that not only involves the natural biological processes of aging, but also the increased risk for different diseases such as coronary heart disease, diabetes and cancer [3]. The primary aging course, itself genetically related, occurs both independently of life style and in the absence of disease [4]. Aging causes structural changes and functional decrements in skeletal muscles (sarcopenia), bone mineral density, cardiovascular and oxygen delivery. Age-related declines are manifest by a decreased ability for aged skeletal muscle to respond to physiological loads such as muscle loading or acute injury. Indeed, older adults often exhibit an age-related reduction in the number and size of muscle fibers, known as sarcopenia [5]. Accordingly, at maximal effort decreases in maximal cardiac output, arteriovenous oxygen difference and maximal oxygen uptake are noted [6,7]. These age modifications are the balance between cellular impairment due to metabolic measures happening inside the cell and offsetting molecular responses that can restore the damage. It is widely accepted that aging is an important risk factor for the development of hypertension and atherosclerosis with its vascular complications. The underlying mechanism whereby aging elevates the probability of disease onset remains obscure; however, it is likely closely related to underlying mechanisms of atherogenesis, the most accepted being the oxidation hypothesis [8]. In brief, a 
vital phase in the progress of atherosclerosis is oxidative alteration of low-density lipoprotein. The oxidation of low-density lipoprotein is a free radical driven lipid peroxidation process and the aldehyde products of lipid hydroperoxide breakdown are responsible for the modification of the low-density lipoprotein apoprotein [9].

Successful aging is a function of both genetic and environmental factors [10]. The primary aging process, which is genetically associated, occurs both independently of life style and in the absence of disease [7]. Aging-related changes occur mainly in the cardiopulmonary and skeletal muscles, bringing about a reduction in physical performance [11]. Maximal work capacity is decreased regardless of lifestyle because of genetic factors. Such consequences contribute to the geriatric syndrome of frailty, thereby severely limiting the function, quality of life and longevity [12].

Muscle mass decreased significantly in aged individuals, after the sixth or seventh decades of life, [13].

This reduction in muscle mass is a major factor in the decline of maximal oxygen uptake, indicating the cardiopulmonary fitness of the individual [14], and therefore, bringing about a reduction in physical work capacity [11]. Such myocardial and peripheral functional changes include a decline in the maximum heart rate, stroke volume, and left ventricular contractility, and an increase in total peripheral resistance. Consequently, oxygen delivery to the working muscle decreases. The human population aged from 0 to 91 years screened previously by ELISA revealed that the level of s-Klotho was related to human physiological declines with aging [15].

\section{Oxidative Stress}

Oxidatively altered low density lipoproteins cause lipidladen macrophage, or foam cell, buildup in the fatty strip, an atherosclerosis process begins. Following employment of immune cells, a pro-inflammatory stage, raises oxidative stress, and starts a sequence of actions including apoptotic vascular and nonvascular cells [16]. It has been suggested that up to $2 \%$ of the oxygen utilized (hyperoxide) in the cell's mitochondria may produce oxygen radicals and peroxisomal level to increase, which may hit and alter DNA, protein, cell membranes and organelles as well as extracellular components [17]. Peroxisomal disorders are inherited diseases representing a group of genetic diseases in humans in which there is a lack of one or more peroxisomal functions [18].

Peroxisomes known as a microbody are organelles containing peroxide enzymes and have distinguishing assignments such as fatty acids breakdown [19]. Peroxisomes are found mainly in the liver and the kidneys, the organs mainly responsible for removing toxic chemicals. Peroxisome carries out oxidation reactions, producing the toxic hydrogen peroxide (H2O2). In addition, peroxisomes hold the enzyme catalase, which causes toxic H2O2 elimination by converting it to $\mathrm{H} 2 \mathrm{O}$ and $\mathrm{O} 2$ [20]. Peroxisomes as well, is important for energy metabolism and is involved in the enzymatic activity of the pentose (5-carbon sugars) phosphate pathway. It is a metabolic pathway producing nicotinamide adenine dinucleotide phosphate (NADPH) and pentoses as well as ribose 5-phosphate, the last one serves as a precursor for the synthesis of nucleotides [21].
Hyperoxide as reactive oxygen species (ROS) is a chemical reactive molecule that contains the superoxide anion, such as peroxides and superoxide [22]. Superoxide is biologically relatively toxic and is arranged by the immune system to eliminate attacking pathogens. Superoxide is produced in large quantities by the enzyme NADPH oxidases [23]. With aging, peroxisomes may boost the production of ROS during cell metabolism Thus, hastening the aging process [24]. In addition, macro-autophagy process declines along aging, resulting in structures changes causing decrease in cell functions abilities [25]. Endothelial dysfunction is usually represented by dysregulation of the arterial normal smooth muscle contractile response, it contributes to the employment of immune cells and the pro-inflammatory stage. Oxidative stress induces endothelial dysfunction, following an elevated in its values, endothelial dysfunction stimulates the atherosclerosis pathological process. In addition, increased oxidative stress is a key factor in the progression of aging-related decreases in cell's normal function [26]. Thus, prooxidant causes atherosclerosis, apoptotic and cell senescence relevant to aging process.

\section{Exercise in aging}

It has been suggested that exercising aged individuals keep high level of endurance capacity [27], meaning; exercising at intensities above the anaerobic threshold. There are growing evidences from; longitudinal and cross-sectional studies that chronic endurance training attenuates the decline in maximal oxygen uptake associated with age [7]. In addition, the position of aerobic exercise as a tool to reduce the risk of numerous long-lasting deteriorating diseases has been convincing recognized earlier [28,29] Also, improved physical work capacity following training may be consider as an accurate indicator of regular physical activity. Improved work capacity includes different physiological system mechanisms in the: cardiopulmonary capacity, body composition, muscular strength and endurance capacity.

Untrained and trained elderly can increase the response of the cardiopulmonary without a significant reduction in peripheral ability to extract oxygen at the muscle level. It was found that in elderly subjects, skeletal muscle mitochondrial capacity, tissue blood flow capacity, and oxygen exchange capacity appear to be well matched. It seems that intrinsic mitochondrial function and regulation are not altered significantly. Therefore, the higher aerobic capacity in the trained elderly is related to increases in the abilities of cardiovascular factors following training and to the lesser extent to increases in muscle mitochondria concentration and capillarity [30].

Both, anaerobic exercise and aerobic exercise generate ROS differently. Increase aerobic exercise intensity along with oxygen uptake results in ROS rise affecting, muscle contractile, and decreases oxidative damage, as long as exercise intensity is up to $75 \%$ [31]. One has to imagine the potential of $2 \%$ of an individual to produce peroxisomal following a fitness session lasting 60 minutes run with an oxygen uptake of 180 LO2. During aerobic exercise ATP production is in the Krebs cycle with oxygen presence, where free fatty acids are the main source of energy production. Anaerobic exercise on the other hand, utilizes the glycolytic 
pathway producing ATP and lactate. Aerobic exercise compared to anaerobic exercise produce larger ROS amount, however, anaerobic exercise induces continually ROS production during recovery. High blood lactate levels possibly has an effect on the increase ROS production by equine polymorphonuclear leukocytes involved in the pathogenesis, which relates to alterations in essential immune functions within a short period after all-out-intensity exercise [32]. Although more oxygen is consumed during aerobic exercise, the generated ROS does not induce significant oxidative damage. Oxygen uptake as such may not be the major cause of exerciseinduced oxidative damage.

Metabolite peroxisome produced by the mitochondria during the energy production process, is modified by aerobic exercise in the redox setting. The peroxisome proliferator activated receptor (PPAR) $\gamma$ coactivator $1-\alpha$ (PGC-1 $\alpha$ ) a lipid catabolism and mitochondrial function regulator [33]. PGC-1 $\alpha$ activation could result in decreased oxidative challenge, either by upregulation of antioxidant enzymes and/or by an increased number of mitochondria that allows lower levels of respiratory activity for the same degree of ATP generation. Usually, Oleic acid a monounsaturated fatty acid, initiates 02- production through NADPH oxidase. However, oleic acid also, can alter cell's reactive oxygen species (ROS) production. Oleic acid slows down electron's sequence of reactions between the cytochromes within the Krebs respiratory chain and thus, due to their protonophoric act on the inner mitochondrial membrane by translocating, protons across lipid bilayers so, it significantly reduces ROS production in the converse mode of electron transport. These effects of oleic acid can modulate signaling functions of ROS, and thus, decrease apoptotic process of cells [34].

The oxidative activity of ROS is a normal function of cellular respiration but can impair the normal functioning of other molecules and systems if present in high amounts [35]. The body has natural antioxidant systems that help to keep ROS levels normal, however, these antioxidant systems can be overwhelmed, causing an acute accumulation of ROS known as oxidative stress [36]. Study by [37] tested the hypothesis that long-lasting aerobic exercise training could prevent the age-associated reduction in s-klotho serum levels, in thirty healthy sportsmen: 15 young aerobically well-trained elite athletes and 15 aerobically well trained master athletes. This study demonstrated that circulating s-Klotho levels are similar for young healthy well-trained elite runners and elite master athletes. This suggests that the response of s-Klotho depends on the aerobic fitness level [5,38]. In addition, levels of s-Klotho were significantly higher in both exercising groups when compared with age matched untrained subjects reported earlier by [39], suggesting that long lasting aerobic training may be an appropriate model for mechanistically probing the role of physical activity on s-Klotho expression.

\section{S-Klotho}

Klotho is a transmembrane protein that, also effects and control over the connection of humans to insulin and seems to be associated to as an anti-aging function [40]. The $\alpha$-Klotho gene is highly preserved in humans and circulates in blood as s-Klotho which acts by way of a humoral factor [41]. In humans, serum levels of s-Klotho decrease with aging progress [42], that may be observed in patients with several aging-related diseases such as coronary artery disease, cancer, hypertension, and kidney disease [43,44]. The klotho gene encodes a single-pass transmembrane protein that binds to multiple fibroblast growth factor receptors and functions as a co-receptor for FGF23, a bone-derived hormone that suppresses phosphate reabsorption and vitamin D biosynthesis in the kidney [45]. In addition, the extracellular domain of s-Klotho protein is shed and secreted [46]. It has previously been demonstrated that Klotho protein increases the ability of cells to remove damaging ROS by increasing the cell functions that detoxify harmful ROS $[47,48]$, however, the precise mechanism of klotho's action is not fully understood, but it changes cellular calcium homeostasis, by both increasing the expression and activity of transient receptor potential cation channels, vanilloid subfamily, member 5 (TRPV5) which plays a key role in active $\mathrm{Ca}++$ reabsorption in the kidney and decreasing that of transient receptor potential canonical 6 (TRPC6), a subtype of calcium-permeable channel [49]. Additionally, Klotho increases membrane expression of the inward rectifier ROMK1 channels i.e. renal outer medullary $\mathrm{K}+[49,50]$.

Klotho-deficient mice show increased production of vitamin D and altered mineral-ion homeostasis is suggested to be a cause of premature aging like phenotypes, because the lowering of vitamin D activity by dietary restriction reverses the premature aging like phenotypes and prolongs survival in these mutants. These results suggest that aging like phenotypes were due to Klotho-associated vitamin D metabolic abnormalities [51,52]. The Klotho protein deficiency is known to participate in prematureaging. Klotho protein contributes in numerous pathways that control aging, for example; phosphate homeostasis, insulin signaling and Wnt signaling regulation [53]. As an aging suppressor, Klotho is an important molecule in aging processes and its overexpression results in longevity [53]. The Klotho gene encodes a transmembrane protein that after cleavage is also found as a secreted protein. However, previously it has been shown that the circulating s-Klotho levels are significantly higher than the membrane-bound form in humans and mice and the latter is significantly higher than the former [53]. Importantly, its overexpression suppresses insulin/IGF-I signaling and thus extends the lifespan. In addition, Klotho participates in the regulation of several other intracellular signaling pathways, including regulation of FGF23 signaling, cAMP, PKC, transforming growth factor- $\beta$ (TGF- $\beta$ ), p53/p21, and Wnt signaling [45,54, 15]. studied, a population-based longitudinal study, revealed that low plasma levels of Klotho are associated with decreased activities of daily living in older individuals. In older community-dwelling adults, plasma klotho is an independent predictor of all-cause mortality [55], Low plasma Klotho levels have also been associated with a poor muscle strength in older, community dwelling adults [56].

\section{Summary}

So far, the present review, suggests that aerobic exercise increase Klotho gene expression, thus may be a biological and harmless tool to slow down the aging process and various agingrelated diseases. In addition, it has been shown that moderate 
aerobic exercise possibly may increase Klotho gene expression in muscle cells and decrease ROD production [56]. However, others suggested lower aerobic intensities as a way to reduce ROS [39]. Following aerobic exercise training, expression of the membranebound Klotho protein levels was suggestively decreased pointing that membrane-bound Klotho protein compared to the secreted form may be the active one.

\section{Conclusion}

The present review suggests that aerobic exercise delays aging process by increase Klotho gene expression, which in turn, reduces ROS damages to the cell. The relationships between the circulating s-Klotho levels a potential anti-aging factor and aerobic exercise are beneficial to physically active aged individuals.

\section{Acknowledgement}

None.

\section{Conflict of Interest}

No conflict of interest.

\section{References}

1. Santos Lozano A, Santamarina A, Pareja Galeano H, Sanchis Gomar F, Fiuza Luces C, et al. (2016) The genetics of exceptional longevity: Insights from centenarians. Maturitas 90: 49-57.

2. Tiffon C (2018) The impact of nutrition and environmental epigenetics on human health and disease. Int J Mol Sci 19(11)

3. Gault ML, Willems ME (2013) Aging, Functional Capacity and Eccentric Exercise Training. Aging Dis 4: 351-363.

4. Dasari V, Srivastava S, Khan S, Mishra RK (2018) Epigenetic factors Polycomb (Pc) and Suppressor of zeste $(\mathrm{Su}(\mathrm{z}) 2)$ negatively regulate longevity in Drosophila melanogaster. Biogerontology 19: 33-45.

5. Keith G Avin, Paul M Coen, Wan Huang, Donna B Stolz Gwendolyn A Sowa, et al. (2014) Skeletal muscle as a regulator of the longevity protein, Klotho Front. Physiol 5: 189.

6. Shephard RJ (1987) Physical Activity and Aging (2nd Ed.). Croom Helm Pp.354.

7. Hawkins S, Wiswell R (2003) Rate and mechanism of maximal oxygen consumption decline with aging: implications for exercise training. Sports Med 33: 877-888.

8. Witztum JL (1994) The oxidation hypothesis of atherosclerosis. Lancet 344: 793-795.

9. Burkitt MJ (2001) A critical overview of the chemistry of copper dependent low-density lipoprotein oxidation: roles of lipid hydroperoxides, alpha-tocopherol, thiols, and ceruloplasmin. Arch Biochem Biophys 394: 117-135.

10. Sebastiani P, Solovieff N, Puca A, Hartley SW, Melista E, et al. (2010) Genetic Signatures of Exceptional Longevity in Humans. Science 333(6041): 404.

11. Thomas L, Levett K, Boyd A, Leung DY, Schiller NB, et al. (2003) Changes in regional left atrial function with aging: evaluation by Doppler tissue imaging. Eur J Echocardiogr 4: 92-100.

12. Afilalo J, Karunananthan S, Eisenberg MJ, Alexander KP, Bergman H (2009) Role of frailty in patients with cardiovascular disease. Am J Cardiol 103: 1616-1621.

13. Chien MY, Kuo HK, Wu YT (2010) Sarcopenia, Cardiopulmonary Fitness, and Physical Disability in Community-Dwelling Elderly People. Phys Ther 90: 1277-1287.

14. Basset DR, Howley ET (2000) Limiting factors for maximum oxygen uptake and determinants of endurance performance. Med Sci Sports Exerc 32: 70-84.
15. Crasto CL, Semba RD, Sun K, Cappola AR, Bandinelli S, et al. (2012) Relationship of low-circulating "anti-aging" klotho hormone with disability in activities of daily living among older community-dwelling adults. Rejuvenation Res 15: 295-301.

16. Patel RP, Darley Usmar VM (1999) Molecular mechanisms of the copper dependent oxidation of low-density lipoprotein. Free Radic Res 30: 1-9.

17. Hancock JT, Desikan R, Neill SJ (2001) Role of Reactive Oxygen Species in Cell Signaling Pathways. Biochem Soc Trans 29: 345-350.

18. Aubourg P, Wanders R (2013) Peroxisomal disorders. Handb Clin Neurol 113: 1593-1609.

19. Wanders RJ, Waterham HR (2006) Biochemistry of mammalian peroxisomes revisited. Ann Rev Biochem 75: 295-332.

20. Gabaldon T (2010) Peroxisome diversity and evolution Philosophical Transactions of the Royal Society of London. Series B Biol Sci 365: 765773.

21. Kruger N J, Von Schaewen A (2003) The oxidative pentose phosphate pathway: structure and organisation. Curr Opin Plant Biol 6: 236-246.

22. Hayyan M, Hashim MA, AlNashef IM (2016) Superoxide Ion: generation and chemical implications. chemical reviews 116: 3029-3085.

23. Frazziano G, Champion HC, Pagano PJ (2012) NADPH oxidase-derived ROS and the regulation of pulmonary vessel tone. Am J Physiol Heart Circ Physiol 302: H2166- H2177.

24. Bergamini E, Cavallini G, Donati A, Gori Z (2004) The role of macroautophagy in the ageing process, anti-ageing intervention and age-associated diseases. Int J Biochem Cell Biol 36: 2392-2404.

25. Donati A (2006) The involvement of macroautophagy in aging and antiaging interventions. Mol Aspects Med 27: 455-470.

26. Finkel T, Holbrook NJ (2000) Oxidants, oxidative stress and the biology of ageing. Nature 408: 239-247.

27. Baldwin KM, Haddad F (2010) Research in the exercise sciences: where we are and where do we go from here--Part II. Exerc Sport Sci Rev 38: 42-50.

28. Joyner JM, Green JD (2009) Exercise protects the cardiovascular system: effects beyond traditional risk factors. J Physiol 587: 5551-5558.

29. Naichun JI, Jing Luan, Fengrui Hu, Yirong Zhao, Bosen Lv, et al. (2018) Aerobic exercise-stimulated Klotho upregulation extends life span by attenuating the excess production of reactive oxygen species in the brain and kidney. Exp Ther Med 16: 3511-3517.

30. Matsakas A, Narkar VA (2010) Endurance exercise mimetics in skeletal muscle. Curr Sports Med Rep 9: 227-232.

31. Matos N, Winsley RJ (2007) Trainability of young athletes and overtraining. J Sports Sci Med 6: 353-67.

32. Echigoya Y, Morita S, Itou T, Sakai T (2012) Effects of extracellular lactate on production of reactive oxygen species by equine polymorphonuclear leukocytes in vitro. Am J Vet Res 73: 1290-1298.

33. Suwa M, Nakano H, Kumagai S (2003) Effects of chronic AICAR treatment on fiber composition, enzyme activity, UCP3, and PGC-1 in rat muscles. J Appl Physiol 95: 960-968.

34. Mishra S, Chattopadhyay A, Naaz S, Ghosh AK, Das AR, et al. (2018) Oleic acid ameliorates adrenaline induced dysfunction of rat heart mitochondria by binding with adrenaline: An isothermal titration calorimetry study. Life Sci 218: 96-111.

35. Mc Bride JM, Kraemer WJ (1999) Free radicals, exercise, and antioxidants. J Strength Cond Res 13: 175-183.

36. Park SY, Kwak YS (2016) Impact of aerobic and anaerobic exercise training on oxidative stress and antioxidant defense in athletes. J Exerc Rehabil 12: 113-117.

37. Saghiv M, Goldhammer E, sagiv M, Ben Sira D (2015) Klotho gene expression responses to long lasting aerobic training and aging. J J Geronto 1(2): 009.

38. Reimers CD, Knapp G, Reimers AK (2012) Does physical activity increase life expectancy? A review of the literature. J Aging Res Pp. 9. 
39. Lee EY, Kim SS, Lee JS, Kim IJ, Song SH, et al. (2014) Soluble $\alpha$-Klotho as a Novel Biomarker in the Early Stage of Nephropathy in Patients with Type 2 Diabetes. Plos One 9(8): e102984.

40. Naichun Ji, Jing Luan, Fengrui Hu, Yirong Zhao, Bosen Lv, et al. (2018) Aerobic exercise-stimulated Klotho upregulation extends life span by attenuating the excess production of reactive oxygen species in the brain and kidney. Exp Ther Med 16: 3511-3517.

41. Rubinek T, Modan Moses D (2016) Klotho and the Growth Hormone/ Insulin-Like Growth Factor 1 Axis: Novel Insights into Complex Interactions. Vitam Horm 101: 85-118.

42. Zhong Zuo, Han Lei, Xiuqing Wang, Yuhong Wang, William Sonntag, et al. (2011) Aging-related kidney damage is associated with a decrease in klotho expression and an increase in superoxide production. Age (Dordr) 33: 261-274.

43. Wang Y, Sun Z (2009) Current understanding of klotho. Ageing Res Rev 8: 43-51.

44. Wang Y, Sun Z (2014) Antiaging gene Klotho regulates endothelin-1 levels and endothelin receptor subtype B expression in kidneys of spontaneously hypertensive rats. Journal of hypertension 32: 16291636.

45. Kuro OM (2008) Klotho as a regulator of oxidative stress and senescence. Biol Chem 389: 233-241.

46. Xiao NM, Zhang YM, Zheng Q, Gu J (2004) Klotho is a serum factor related to human aging. Chin Med J (Engl) 117: 742-747.

47. Baluchnejadmojarad T, Eftekhari SM, Jamali Raeufy N, Haghani S, Zeinali H, et al. (2017) The anti-aging protein klotho alleviates injury of nigrostriatal dopaminergic pathway in 6-hydroxydopamine rat model of Parkinson's disease: Involvement of PKA/CaMKII/CREB signaling. Exp Gerontol 10: 70-76.
48. Guo Y, Zhuang X, Huang Z, Zou J, Yang D, et al. (2018) Klotho protects the heart from hyperglycemia-induced injury by inactivating ROS and NF$\kappa \mathrm{B}$-mediated inflammation both in vitro and in vivo. Biochim Biophys Acta Mol Basis Dis 1864: 238-251.

49. Huang CL (2010) Regulation of ion channels by secreted Klotho: mechanisms and implications. Kidney Int 77: 855-860.

50. Liu BC, Yang LL, Lu XY, Song X, Li XC, et al. (2015) Lovastatin-Induced Phosphatidylinositol-4-Phosphate 5-Kinase Diffusion from Microvilli Stimulates ROMK Channels. J Am Soc Nephrol 26: 1576-1587.

51. Medici D, Razzaque MS, Deluca S, Rector TL, Hou B, et al. (2008) FGF23-Klotho signaling stimulates proliferation and prevents vitamin D-induced apoptosis. J Cell Biol 182: 459-465.

52. Kuro OM. Klotho and aging. Biochim Biophys Acta 1790: 1049-1058.

53. Mencke R, Hillebrands JL, NIGRAM consortium (2017) The role of the anti-ageing protein Klotho in vascular physiology and pathophysiology. Ageing Res Rev 35: 124-146.

54.Sopjani M, Rinnerthaler M, Kruja J, Dermaku Sopjani M (2015) Intracellular signaling of the aging suppressor protein Klotho. Curr Mol Med 15: 27-37.

55. Semba RD, Cappola AR, Sun K, Bandinelli S, Dalal M, et al. (2011) Plasma klotho and mortality risk in older community-dwelling adults. J Gerontol A Biol Sci Med Sci 66: 794-800.

56. Semba RD, Cappola AR, Sun K, Bandinelli S, Dalal M, et al. (2012) Relationship of low plasma klotho with poor grip strength in older community-dwelling adults: the InCHIANTI study. Eur J Appl Physiol 112: $1215-1220$.

57. Skulachev MV, Skulachev VP (2017) Programmed aging of mammals: Proof of concept and prospects of biochemical approaches for anti-aging therapy. Biochemistry (Mosc) 82: 1403-1422. 